\title{
ESTUDO DO EQUILÍBRIO E CINÉTICA DA BIOSSORÇÃO DO Pbº POR Saccharomyces cerevisiae
}

\author{
Joelma Morais Ferreira, Flávio Luiz Honorato da Silva*, Odelsia Leonor Sanchez Alsina, Líbia de Sousa Conrado \\ Oliveira, Eliane Bezerra Cavalcanti e Wolia Costa Gomes \\ Unidade Acadêmica de Engenharia Química, Centro de Ciências e Tecnologia, Universidade Federal de Campina Grande, \\ Av. Aprígio Veloso, 882, 58109-970 Campina Grande - PB, Brasil
}

Recebido em 12/7/06; aceito em 1/12/06; publicado na web em 24/7/07

\begin{abstract}
EQUILIBRIUM AND KINETIC STUDY OF Pb ${ }^{2+}$ BIOSORPTION BY Saccharomyces cerevisiae. The biosorption, based on the use of biomass for removal of ions is distinguished as an innovative and promising technology when compared with the traditional methods. In this context, the aim of the present work is to use Saccharomyces cerevisiae as biosorbent for the retention of $\mathrm{Pb}^{2+}$ metal ions. Factorial design was used for evaluation of the process. The observed equilibrium data were well described by Langmuir and Freundlich adsorption isotherms. The maximum adsorption capacity was $1486.88 \mathrm{mg} / \mathrm{g}$. The results indicated that Saccharomyces cerevisiae is suitable for biosorption of $\mathrm{Pb}^{2+}$ metal ions.
\end{abstract}

Keywords: heavy metal; experimental design; Langmuir isotherm.

\section{INTRODUÇÃO}

As atividades industriais têm introduzido metais nas águas em uma quantidade muito maior que aquela que seria natural, causando grandes poluições. Os tratamentos convencionais (redução química, troca iônica, ultrafiltração e osmose inversa), normalmente usados para a remoção de metais dos efluentes líquidos, apresentam algumas desvantagens, pois além de serem, na maioria, processos caros não conseguem remover totalmente os íons metálicos, gerando ainda substâncias tóxicas que necessitam serem eliminadas cuidadosamente, em mais outra etapa de processo ${ }^{1-5}$.

$\mathrm{O}$ chumbo é encontrado na natureza acumulado em minas como resultado dos processos de diferenciação que ocorreram durante a evolução do planeta. Os resíduos de chumbo são classificados pelas normas brasileiras como perigosos. A exposição ao chumbo utilizado em diversos tipos de indústrias (mineração, cerâmica, baterias, pintura) é cada vez mais comum e os desastres são descobertos apenas anos ou décadas mais tarde. $\mathrm{O}$ contato humano com o chumbo pode levar a distúrbios de praticamente todas as partes do organismo - sistema nervoso central, sangue e rins - culminando com a morte. Em doses menores, acarreta alteração na produção de hemoglobina e em processos bioquímicos cerebrais causando alterações psicológicas e comportamentais. Devido a sua baixa solubilidade, a absorção dá-se principalmente por via oral ou respiratória.

A biossorção é baseada na utilização de biomassas microbianas como bactérias, fungos e algas. Destaca-se como uma grande alternativa para remoção de metais pois, quando comparada com os processos convencionais, apresenta reconhecidas vantagens como a biomassa pode ser reutilizada; os metais podem ser removidos da solução independentemente do grau de toxidez; os tempos de operação são pequenos quando o equilíbrio é alcançado; não produz compostos secundários com toxicidade e pode ser altamente seletiva ${ }^{6-9}$.

Vários microrganismos já foram estudados na remoção de íons metálicos. Pagnanelli et al. ${ }^{10}$ estudaram a biossorção dos íons metálicos $\mathrm{Pb}, \mathrm{Cu}, \mathrm{Zn}$ e Cd através da bactéria Sphaerotilus natans e mostraram que a biomassa possuía capacidade de remoção desses íons. Nourbakhsh et al. ${ }^{11}$ pesquisaram a biossorção de vários íons

*e-mail: flavioluizh@yahoo.com.br metálicos $\left(\mathrm{Cr}^{6+}, \mathrm{Pb}^{2+}\right.$ e $\left.\mathrm{Cu}^{2+}\right)$ pelo Bacillus sp. Outro tipo de bactéria testada foi Streptomyces rimosus ${ }^{7}$, que adsorveu aproximadamente $137 \mathrm{mg} / \mathrm{g}$ de $\mathrm{Pb}^{2+}$. Muitas espécies de fungos, como o Neurospora crassa com capacidade adsortiva de 49,06 mg/g para íons de chumbo ${ }^{12}$ e Mucor rouxii com capacidade de remoção de íons $\mathrm{Pb}^{2+}$ equivalente a $35,69 \mathrm{mg} / \mathrm{g}^{13}$, também já foram utilizadas como biomassa em processos de biossorção. Entre os fungos, as leveduras são as mais exploradas cientificamente, devido ao fato de serem organismos eucarióticos mais facilmente manipulados e, assim, servirem de excelente modelo para o estudo.

A Saccharomyces cerevisiae é utilizada na produção de álcool etílico por fermentação, do qual o Brasil produz cerca de 13 bilhões de L por ano, como também em panificação; é também conhecida por acumular grandes quantidades de metais em meios aquosos.

Sendo o Brasil o maior produtor mundial de álcool etílico via processo fermentativo utilizando-se da Saccharomyces cerevisiae (levedura) como o microrganismo agente da fermentação, é prática comum nas indústrias de produção de álcool etílico a sangria do creme de levedura, que consiste em retirar parte do creme do processo de centrifugação. Desta forma, a Saccharomyces cerevisiae é uma fonte excedente do processo de fermentação, que pode ser utilizada, por exemplo, como bioadsorvente em processos de separação física. A levedura Saccharomyces cerevisiae possui capacidade de retirar metais da água, podendo ser usada como bioacumulador desses metais, sendo uma ótima alternativa para a descontaminação ambiental ${ }^{14,15}$.

O presente trabalho teve como objetivos estudar o equilíbrio e a cinética, em meio estático, da remoção de chumbo $\left(\mathrm{Pb}^{2+}\right)$ avaliando os efeitos das variáveis: concentração inicial de metal, temperatura, concentração de biomassa, $\mathrm{pH}$ e estado da biomassa, em soluções aquosas sintéticas, através da biossorção utilizando leveduras (Saccharomyces cerevisiae)

\section{Isotermas de adsorção}

A adsorção pode ser avaliada quantitativamente através de isotermas, as quais permitem avaliar a capacidade de adsorção e a força com a qual os metais se ligam a determinado adsorvente ${ }^{16}$.

Uma isoterma de adsorção mostra a quantidade de um determinado soluto adsorvido por uma superfície adsorvente, em função da con- 
centração de equilíbrio do soluto ${ }^{17,18}$. Para se obter uma isoterma, coloca-se em contato a solução contendo o componente a ser adsorvido a diferentes concentrações iniciais e temperatura constante até atingir o equilíbrio, determinando-se, assim, a quantidade de material adsorvido. O comportamento gráfico das isotermas pode apresentarse de várias formas, fornecendo informações importantes sobre o mecanismo de adsorção, através de parâmetros de ajustes obtidos de modelos matemáticos que os representam. $\mathrm{Na}$ adsorção, o pH, a temperatura e, principalmente, o tipo de adsorvente são parâmetros que influenciam na forma da isoterma. As equações que representam o comportamento das isotermas de adsorção comumente usadas são: Langmuir, Freundlich, Henry e Brunauer, Emmett, Teller (BET) ${ }^{19}$.

\section{Isoterma de Langmuir}

A teoria de Langmuir assume que todas as forças que atuam na adsorção são similares em natureza àquelas que envolvem uma reação química, e que a sorção se resume a uma única camada de moléculas da substância sobre a superfície das partículas sólidas, sendo as forças atrativas desenvolvidas entre o sorvente e o sorvido, essencialmente de curta duração ${ }^{20}$. Esta isoterma pode ser representada pela Equação 1 .

$$
\frac{\mathrm{q}}{\mathrm{q}_{\mathrm{m}}}=\frac{\mathrm{KC}}{1+\mathrm{KC}}
$$

onde q é a concentração de sorbato; $\mathrm{q}_{\mathrm{m}}$ é capacidade máxima de adsorção; $\mathrm{K}$ é a constante de equilíbrio relacionada com a energia livre de adsorção, que corresponde à afinidade entre a superfície do adsorvente e o soluto e $\mathrm{C}$, a concentração de equilíbrio.

Apesar das limitações contidas nas hipóteses de Langmuir, a sua equação ajusta-se razoavelmente bem aos dados experimentais de muitos sistemas ${ }^{21}$.

\section{Isoterma de Freundlich}

A isoterma de Freundlich corresponde a uma distribuição exponencial de calores de adsorção. Os sistemas reais podem ser mais bem representados por este tipo de isoterma, que é expressa pela Equação 2

$\mathrm{q}=\mathrm{KC} \mathrm{C}^{1 / \mathrm{n}}$

onde K e n são constantes que dependem de diversos fatores experimentais e se relacionam com a distribuição dos sítios ativos e a capacidade de adsorção de líquidos.

\section{PARTE EXPERIMENTAL}

\section{Biomassa}

Nesse trabalho foi utilizado, como biomassa, o microrganismo Saccharomyces cerevisiae, proveniente do fermento comercial da Fleischmann Royal ${ }^{\circledR}$ com umidade de $72 \%$ (base úmida) e proteína bruta igual a $45 \%$ (base seca).

\section{Estudo cinético}

Para determinar o tempo de contato necessário para que o equilíbrio entre a biomassa e a solução de metal fosse atingido, foi realizado o estudo da cinética de biossorção do íon metal $\mathrm{Pb}^{2+}$ pela levedura Saccharomyces cerevisiae. Essa etapa foi realizada em erlenmeyers de $250 \mathrm{~mL}$ contendo $100 \mathrm{~mL}$ de soluções do metal mais $0,01 \mathrm{~g}$ da biomassa in natura em base úmida, o que corresponde a $0,0028 \mathrm{~g}$ em base seca. As concentrações utilizadas para o chumbo $\left(\mathrm{Pb}^{2+}\right)$ variaram entre 0,282 e $3,4 \mathrm{mg} / \mathrm{L}$. Os erlenmeyers foram mantidos, sem agitação, a uma temperatura de $30^{\circ} \mathrm{C}$ (estudo cinético estático).

As amostras foram preparadas em duplicatas e as alíquotas das soluções do metal foram retiradas em diferentes intervalos de tempo $(24,48,72$ e $96 \mathrm{~h})$. As alíquotas foram centrifugadas a uma velocidade de $1000 \mathrm{rpm}$ por aproximadamente $10 \mathrm{~min}$ e analisadas por técnica de polarografia ASV usando o polarógrafo modelo POL 150 acoplado a uma célula de mercúrio modelo MDE 150, ambos da marca Radiometer - Analytical.

A quantidade de íons metálicos adsorvida pela levedura foi calculada através da Equação 3.

$q=\frac{V\left(C_{i}-C_{f}\right)}{M}$

onde $\mathrm{C}_{\mathrm{i}}$ é a concentração inicial do metal, $\mathrm{C}_{\mathrm{f}}$ a concentração de equilíbrio do metal, $\mathrm{M}$ é a massa do biossorvente (base seca) e $\mathrm{V}$ o volume da solução de íons metálicos.

Estudo da influência da quantidade de biomassa, concentração inicial do metal na solução e temperatura sobre o processo

A biossorção depende de parâmetros como concentração do íon, concentração de biomassa e temperatura ${ }^{19}$.

A influência das variáveis quantidade de biomassa em base úmida (B), concentração inicial de metal $(\mathrm{C})$ e temperatura (T) foi estudada utilizando-se a técnica de planejamento fatorial. Nesse estudo foram escolhidos os níveis para cada variável, conforme os seguintes critérios: os valores de concentração inicial de metal foram superiores aos estabelecidos pelo CONAMA (resolução 357), com o intuito de abranger uma faixa suficientemente larga, de modo a contemplar diversos efluentes encontrados na indústria, como por exemplo, efluentes de indústrias de baterias que possuem níveis altíssimos de chumbo, como é o caso do Grupo Moura com sede em Pernambuco (Brasil), onde já foi encontrada concentração de $4,9 \mathrm{mg} / \mathrm{L}$ desse metal tóxico ${ }^{22}$. O intervalo de temperatura representa os extremos encontrados na região Nordeste. Quanto aos níveis de concentração de biomassa, foram definidos com base em dados obtidos na literatura ${ }^{7}$. Os valores de cada nível são apresentados na Tabela 1 .

Tabela 1. Níveis das variáveis do planejamento fatorial completo $2^{3}$ para o chumbo

\begin{tabular}{lccc}
\hline Variáveis & Nível $(-1)$ & Ponto central (0) & Nível $(+1)$ \\
\hline $\mathrm{B}$ & 0,01 & 0,5 & 1 \\
$\mathrm{C}$ & 0,44 & 2,97 & 5,5 \\
$\mathrm{~T}$ & 27 & 35 & 43 \\
\hline
\end{tabular}

O estudo do equilíbrio também foi realizado por método estático, utilizando-se efluentes sintéticos para determinação do efeito da concentração do metal na capacidade de biossorção.

As condições experimentais adotadas para a obtenção das isotermas foram: temperatura constante em $30^{\circ} \mathrm{C}, \mathrm{pH} 6$, concentração de biomassa (morta) igual a $0,0028 \mathrm{~g}$ (base seca) e as concentrações iniciais do íon metálico variaram de 0,36 a $500 \mathrm{mg} / \mathrm{L}$. As soluções sintéticas foram preparadas com água deionizada e uma solução de chumbo padrão titrisol (Merck). A solução (metal mais a biomassa) foi mantida em estufa por $72 \mathrm{~h}$. As concentrações finais do íon metálico presentes nas amostras foram analisadas e 
quantificadas com o auxílio do Polarógrafo POL 150 acoplado a uma célula de mercúrio modelo MDE 150, ambos da marca Radiometer - Analytical.

\section{RESULTADOS E DISCUSSÃO}

\section{Cinética da biossorção do chumbo através da Saccharomyces cerevisiae}

O tempo de contato necessário para que o equilíbrio entre as fases seja atingido é função de vários fatores, entre eles o tipo de biomassa (quantidade e tipos de sítios envolvidos na biossorção), tamanho e forma da biomassa, estado da biomassa (ativa ou inativa, livre ou imobilizada), além do metal envolvido no sistema de biossorção. Para saber o período em que o sistema possa alcançar o equilíbrio foi necessário fazer um estudo cinético. O objetivo deste estudo foi determinar o tempo de contato necessário para que o equilíbrio entre a biomassa e a solução de metal fosse atingido.

A Figura 1 apresenta os resultados obtidos para a biomassa em duas concentrações iniciais distintas.

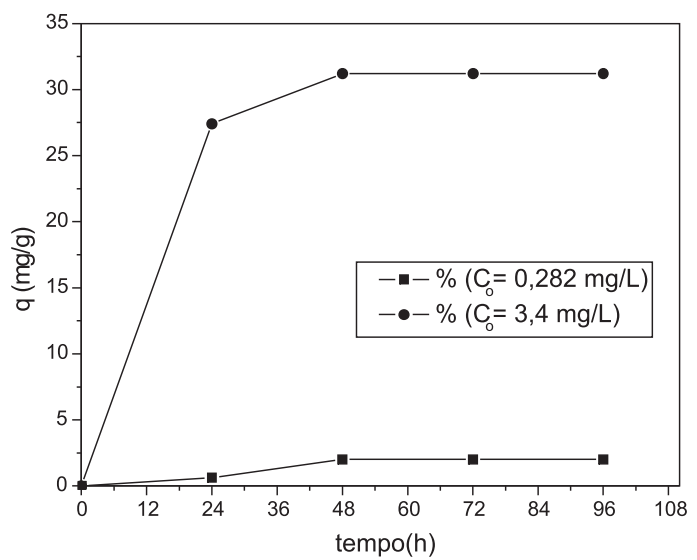

Figura 1. Cinética de biossorção do chumbo pela levedura Saccharomyces cerevisiae $\left(T=30^{\circ} \mathrm{C}\right)$

É possível verificar preliminarmente pelos resultados obtidos que, independentemente da concentração inicial, o tempo necessário para atingir o equilíbrio em condições estáticas foi de 48 h. O tempo de contato de $72 \mathrm{~h}$, definido na metodologia para o levantamento das isotermas, foi mais que suficiente para que o sistema alcançasse o equilíbrio. Embora alguns processos industriais utilizem condições estáticas, a grande maioria dos sistemas de tratamento de efluentes opera em condições dinâmicas, onde os tempos de processamento são bem menores.

\section{Efeito da quantidade de biomassa, concentração inicial do metal na solução e temperatura}

Com a utilização do planejamento experimental fatorial foi possível realizar um estudo mais abrangente das variáveis independentes, realizando de maneira mais organizada uma quantidade mínima de experimentos ${ }^{23}$. Foram realizados 11 ensaios no planejamento experimental para o metal em estudo, sendo 3 deles repetições no ponto central.

A Tabela 2 apresenta os resultados obtidos do planejamento com a resposta (quantidade adsorvida) em cada combinação de níveis para as variáveis quantidade de biomassa, concentração de metal e temperatura.

A partir dos resultados obtidos no planejamento foi realizada uma regressão não linear dos dados experimentais. Sua aplicação
Tabela 2. Matriz do planejamento experimental fatorial $2^{3}$

\begin{tabular}{lccc}
\hline $\begin{array}{l}\text { Quantidade de } \\
\text { Biomassa (B) }\end{array}$ & $\begin{array}{c}\text { Concentração de } \\
\text { metal (C) }\end{array}$ & $\begin{array}{c}\text { Temperatura } \\
(\mathrm{T})\end{array}$ & $\begin{array}{c}\mathrm{q}(\mathrm{mg} / \mathrm{g} \text { de } \\
\text { levedura) }\end{array}$ \\
\hline+1 & +1 & +1 & 1,830 \\
+1 & +1 & -1 & 1,960 \\
+1 & -1 & +1 & 0,160 \\
+1 & -1 & -1 & 0,053 \\
-1 & +1 & +1 & 160,14 \\
-1 & +1 & -1 & 159,46 \\
-1 & -1 & +1 & 15,71 \\
-1 & -1 & -1 & 12,64 \\
0 & 0 & 0 & 1,390 \\
0 & 0 & 0 & 1,460 \\
0 & 0 & 0 & 1,920 \\
\hline
\end{tabular}

permite selecionar a combinação de níveis maximizados na obtenção da melhor resposta para cada situação.

O modelo codificado da regressão dos dados experimentais para o chumbo está representado na Equação 4, onde os parâmetros em negrito são os valores estatisticamente significativos, em nível de 95\% de confiança. Observa-se no modelo os desvios padrão para cada coeficiente.

$\mathrm{q}=(32,28 \pm 9,44)-(43,2 B \pm 11,07)+(36,65 C \pm 11,07)-(36,2 B C$

$\pm \mathbf{1 1 , 0 7})-(0,68 \mathrm{BT} \pm 11,02)-(0,53 \mathrm{CT} \pm 11,02)$

Na Tabela 3 encontram-se os parâmetros da análise de variância (ANOVA).

Tabela 3. Análise da variância para o ajuste do modelo linear (ANOVA)

\begin{tabular}{lc}
\hline \% variância explicada & 90,30 \\
Coeficiente de correlação & 0,950 \\
Teste F (calculado) & 9,30 \\
F tabelado para 95\% de confiança & 5,05 \\
F calculado/F tabelado & 1,84 \\
\hline
\end{tabular}

Pela Tabela 3, observa-se que o coeficiente de correlação foi de aproximadamente 0,95 . Com relação ao teste $\mathrm{F}$, verifica-se que o modelo é estatisticamente significativo em nível de $95 \%$ de confiança, pois o $\mathrm{F}_{\text {calculado }}$ foi maior que $0 \mathrm{~F}_{\text {tabelado }}{ }^{23}$.

As superfícies de resposta foram construídas para todas as possíveis combinações de variação de duas variáveis, mantendo-se a outra fixada. Nas Figuras 2 e 3 são apresentados os gráficos referentes às superfícies de resposta otimizadas, onde a quantidade de biomassa utilizada foi de $0,01 \mathrm{~g}$ (in natura) e a concentração de íons de chumbo foi equivalente a $5,5 \mathrm{mg} / \mathrm{L}$, do planejamento realizado para o metal em estudo.

Através das Figuras 2 e 3 pode-se verificar que com a diminuição da quantidade de biomassa e aumento da concentração inicial do metal aumenta-se a quantidade adsorvida de metal, para qualquer temperatura, obtendo-se valores de q de até $160 \mathrm{mg} / \mathrm{g}$ em base úmida, nas condições operacionais estudadas neste trabalho.

\section{Efeito do pH e estado da biomassa na biossorção}

$\mathrm{O}$ efeito do $\mathrm{pH}$ e as condições da biomassa podem afetar a sorção dos íons metálicos. A diferença entre a biossorção da biomassa morta ou viva pode ser evidenciada na toxidez das células microbianas provocada pela presença dos íons metálicos ${ }^{16}$. Portanto, é importante estudar o estado da biomassa (viva ou morta) em função das diferenças na estrutura e composição das paredes 


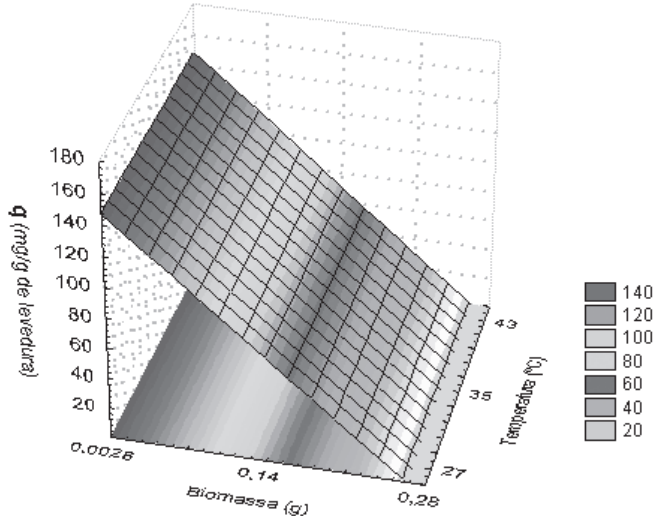

Figura 2. Efeito da quantidade de biomassa e da temperatura sobre a quantidade adsorvida
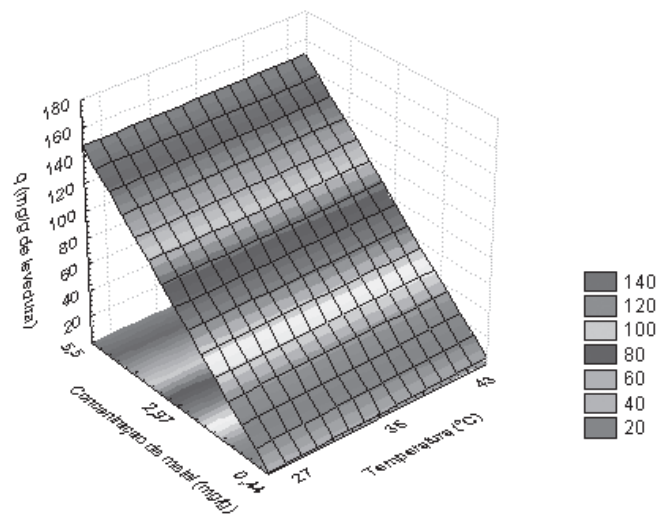

Figura 3. Efeito da concentração do metal e da temperatura sobre a quantidade adsorvida

celulares dos biossorventes, visto que interfere no processo de biossorção. $\mathrm{O}$ pH ótimo de adsorção é diferente para cada metal e microrganismo estudado. Nesse sentido, foi realizado um estudo com auxílio do planejamento experimental fatorial para verificar o efeito do $\mathrm{pH}$ e da viabilidade da biomassa (morta e viva) no processo de biossorção do íon de chumbo.

Na Tabela 4 encontram-se os resultados obtidos no planejamento fatorial elaborado, para o íon chumbo, com a resposta (quantidade adsorvida) em cada combinação de níveis das variáveis de entrada.

Tabela 4. Matriz do planejamento experimental fatorial $2^{2}$

\begin{tabular}{lcc}
\hline $\mathrm{pH}$ & $\mathrm{B}$ & $\begin{array}{c}\mathrm{q} \text { (mg de íons metálicos/ } \\
\mathrm{g} \text { de levedura) }\end{array}$ \\
\hline$-1(2)$ & -1 (morta) & 12,23 \\
$+1(6)$ & -1 (morta) & 119,93 \\
$-1(2)$ & +1 (viva) & 138,48 \\
$+1(6)$ & +1 (viva) & 115,43 \\
$-1(2)$ & -1 (morta) & 6,035 \\
$+1(6)$ & -1 (morta) & 119,31 \\
$-1(2)$ & +1 (viva) & 124,16 \\
$+1(6)$ & +1 (viva) & 116,31 \\
\hline
\end{tabular}

Com os dados obtidos de q através do planejamento fatorial realizou-se uma regressão dos dados experimentais. A Equação 5 apresenta o modelo codificado da regressão dos dados experimentais para o chumbo nas condições estudadas. Estão apresentados nessa equação apenas os parâmetros estatisticamente significativos e os desvios padrão, em nível de $95 \%$ de confiança. $\mathrm{q}=(93,99 \pm 1,95)+(23,76 \mathrm{pH} \pm 1,95)+(29,61 \mathrm{~B} \pm 1,95)-$ $(31,48 \mathrm{pHB} \pm 1,95)$

A Tabela 5 mostra os parâmetros da análise de variância (ANOVA).

Tabela 5. Análise da variância para o ajuste do modelo linear (ANOVA) para o $\mathrm{Pb}^{+2}$

$\begin{array}{lc}\text { \% variância explicada } & 99,37 \\ \text { Coeficiente de correlação } & 0,997 \\ \text { Teste F (calculado) } & 212,20 \\ \text { F tabelado para 95\% de confiança } & 6,59 \\ \text { F calculado/F tabelado } & 32,20\end{array}$

Observando os valores encontrados na Tabela 5, verifica-se que de acordo com o teste $\mathrm{F}$, o modelo é estatisticamente significativo em nível de $95 \%$ de confiança e é altamente preditivo, pois o $\mathrm{F}$ foi bem maior que o $\mathrm{F}_{\text {tabelado }}$

Sendo o modelo codificado (Equação 5) estatisticamente significativo, podem-se construir superfícies de resposta para verificar as faixas das variáveis de entrada em estudo que maximizem a quantidade adsorvida dos íons metálicos.

A superfície de resposta do planejamento fatorial experimental $2^{2}$ realizado está apresentada na Figura 4 onde se observa que, a maior quantidade adsorvida de chumbo (aproximadamente $150 \mathrm{mg}$ / g) pela Saccharomyces cerevisiae se encontra na região onde o $\mathrm{pH}$ é igual a 2 e com a biomassa viva.

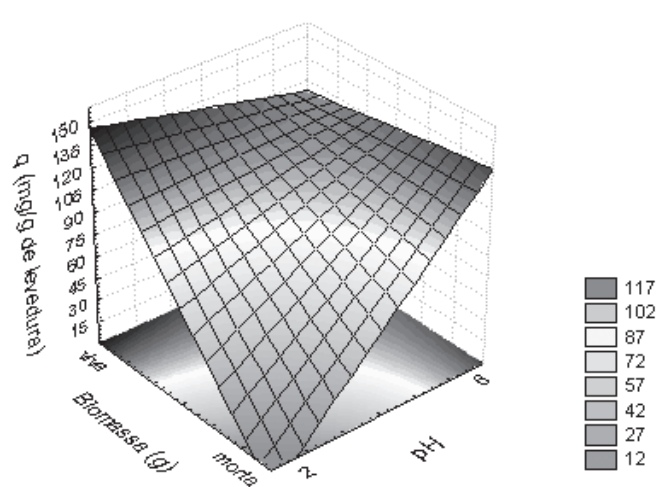

Figura 4. Efeito do pH e tipo de biomassa sobre a quantidade adsorvida

\section{Levantamento da isoterma de adsorção do metal}

Diante dos resultados obtidos, foi possível verificar claramente que quanto menor a quantidade de biomassa, maior a quantidade adsorvida do íon metálico por unidade de massa do adsorvente, ou seja, um aumento no valor de q. Foi observado também que a levedura Saccharomyces cerevisiae apresenta uma boa capacidade de adsorver o $\mathrm{Pb}^{2+}$, quando comparada com outros tipos de biomassas já estudadas e encontradas na literatura, como Streptomyces rimosus que adsorveu cerca de 137 mg/g ${ }^{10}$, Arthrobacter sp. adsorvendo $130 \mathrm{mg} / \mathrm{g}^{20}$ e Mucor rouxii com capacidade máxima de adsorção de $35,69 \mathrm{mg} / \mathrm{g}^{15}$.

As variáveis concentração inicial do íon chumbo, temperatura e quantidade de biomassa apresentaram mais influência sobre a quantidade adsorvida que as variáveis estudadas no último planejamento ( $\mathrm{pH}$ e melhor estado da levedura). Ou seja, para qualquer valor de $\mathrm{pH}$ e estado da levedura não houve aumento na quantidade adsorvida do íon metal em estudo, fixando-se as variáveis quantidade de biomassa e concentração inicial do íon chumbo, pois essas 
Tabela 6. Parâmetros dos modelos de Langmuir e Freundlich

\begin{tabular}{|c|c|c|c|c|c|c|}
\hline & \multicolumn{3}{|c|}{ Langmuir } & \multicolumn{3}{|c|}{ Freundlich } \\
\hline & $\mathrm{q}_{\mathrm{m}}(\mathrm{mg} / \mathrm{g})$ & $\mathrm{B}(\mathrm{L} / \mathrm{mg})$ & $\mathrm{R}^{2}$ & $\mathrm{~K}^{\prime}(\mathrm{L} / \mathrm{g})$ & $1 / \mathrm{n}$ & $\mathrm{R}^{2}$ \\
\hline Modelo Linear & 1233,00 & 0,0094 & 0,91 & 30,6 & 0,57 & 0,95 \\
\hline Modelo Não Linear & 1486,88 & 0,0055 & 0,98 & 52,24 & 0,50 & 0,95 \\
\hline
\end{tabular}

variáveis apresentam influências (efeitos sobre a quantidade adsorvida) muito superiores às outras variáveis.

Desta forma, Ferreira et al. ${ }^{24}$, observaram que utilizando a levedura morta e $\mathrm{pH} 6$ o íon metal chumbo era melhor adsorvido pela levedura Saccharomyces cerevisiae. Assim, o estudo de equilíbrio (isoterma de adsorção) do chumbo foi realizado fixando-se a quantidade de biomassa inativa em $0,01 \mathrm{~g}$ (base úmida), o que corresponde a 0,0028 g (base seca). O sistema em batelada foi mantido a uma temperatura de $35{ }^{\circ} \mathrm{C}, \mathrm{pH} 6$ e a biomassa inativa.

Os modelos de isotermas utilizados nos experimentos para representar os dados de equilíbrio foram os de Langmuir e Freundlich. Os dados de equilíbrio dos sistemas de biossorção sobre o chumbo e as respectivas curvas dos modelos das isotermas estão ilustrados nas Figuras 5 e 6.

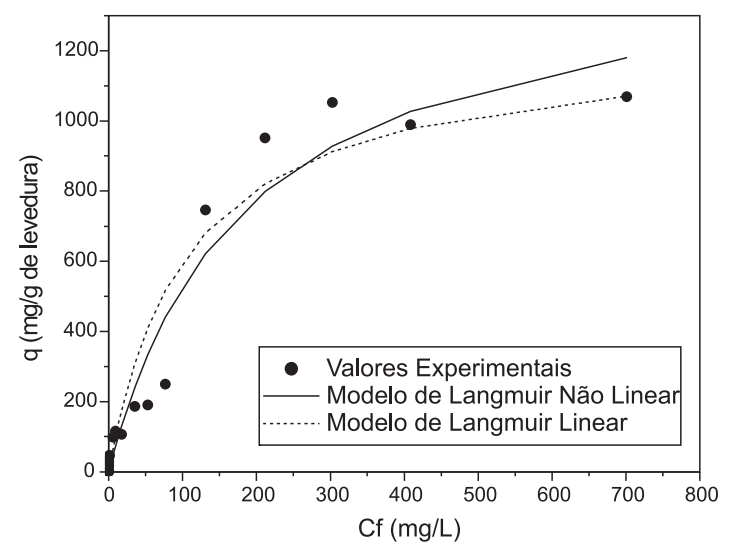

Figura 5. Isotermas de adsorção do íon $\mathrm{Pb}^{2+}$ ajustadas pelo modelo de Langmuir ( $\mathrm{C}=0,36$ a $500 \mathrm{mg} / \mathrm{L}, \mathrm{T}=30{ }^{\circ} \mathrm{C}, \mathrm{pH}$ 6) utilizando a levedura morta

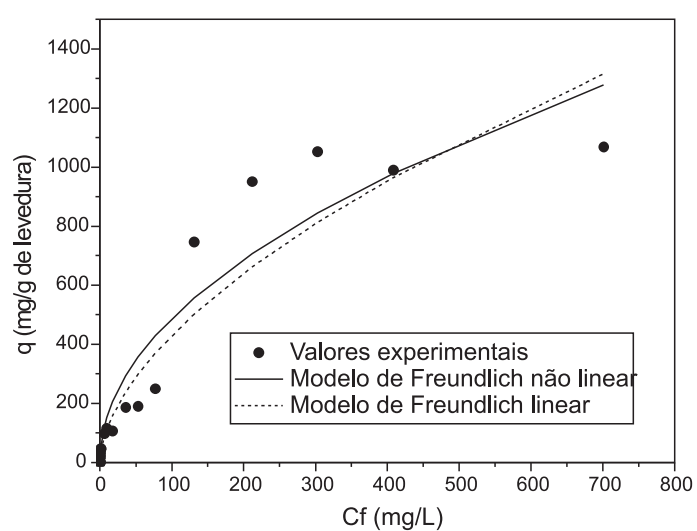

Figura 6. Isotermas de adsorção do íon $\mathrm{Pb}^{2+}$ ajustadas pelo modelo de Freundlich $\left(\mathrm{C}=0,36\right.$ a $500 \mathrm{mg} / \mathrm{L}, \mathrm{T}=30^{\circ} \mathrm{C}, \mathrm{pH}$ 6) utilizando a levedura morta

A Tabela 6 apresenta os valores das constantes do modelo de Langmuir $\left(\mathrm{q}_{\mathrm{m}}\right.$ e b) e do modelo de Freundlich ( $\mathrm{K}^{\prime}$ e $\left.1 / \mathrm{n}\right)$, que refletem convenientemente a natureza do material adsorvente e podem ser usados para comparar o desempenho da biossorção. O parâmetro 1/n encontrado na Equação de Freundlich mede a intensidade pela qual a biomassa adsorve os íons metálicos. Quanto maior o valor de $1 / \mathrm{n}$ maior a tendência dos valores experimentais se ajustarem ao modelo de Freundlich, como pode ser observado na literatura com os íons $\mathrm{Co}^{2+}$ e $\mathrm{Al}^{2+}$, que apresentaram valores de 1,01 e 1,64, respectivamente, bem superiores ao valor encontrado neste trabalho, dados experimentais que foram melhores ajustados por esse modelo, utilizando como biomassa a Telfairia occidentalis ${ }^{25}$. Na Tabela 6 também encontram-se os coeficientes de correlação obtidos para os dois modelos utilizados. Os parâmetros foram obtidos pela regressão linear e não linear dos dados experimentais, utilizando-se do programa Statistica, versão 5.0. O modelo de Freundlich não ajusta bem os dados experimentais, observando-se os valores de coeficiente de correlação e o parâmetro 1/n. Pode-se observar, através dos valores dos coeficientes de regressão $\left(\mathrm{R}^{2}\right)$, que o modelo da isoterma de adsorção de Langmuir obteve melhor ajuste.

Para o ajuste dos dados experimentais usando o modelo de Langmuir com regressão não linear, a capacidade máxima $\left(\mathrm{q}_{\mathrm{m}}\right)$ dos íons de chumbo adsorvidos pela Saccharomyces cerevisiae foi de $1486,88 \mathrm{mg} / \mathrm{g}$, enquanto com ajuste dos dados com regressão linear, obteve-se $\mathrm{q}_{\mathrm{m}}$ de $1233,00 \mathrm{mg} / \mathrm{g}$. Observa-se que, para quaisquer das regressões, o valor de $\mathrm{q}_{\mathrm{m}}$ é bem superior quando comparado com outros tipos de biomassa encontradas na literatura como, por exemplo, os fungos Streptomyces rimosus ${ }^{7}$, Mucor rouxi ${ }^{21}$ e Bacillus firmus $^{20}$ nos quais adsorveram aproximadamente 137, 769 e 1103 $\mathrm{mg} / \mathrm{g}$, respectivamente, utilizando o modelo de Langmuir.

Verifica-se que a regressão linear dos dados usando o modelo de Langmuir apresenta valor de capacidade máxima de adsorção $\left(\mathrm{q}_{\mathrm{m}}\right)$ mais adequado quando se compara com os dados experimentais, ou seja, observando-se a Figura 5, verifica-se que os 4 últimos dados experimentais de q (quantidade adsorvida), valores acima de $200 \mathrm{mg} / \mathrm{L}$ de concentração de equilíbrio, estão praticamente no patamar de saturação, sendo em média de 1050,00 mg/g, existindo mais concordância com o $\mathrm{q}_{\mathrm{m}}$ de 1233,00 mg/g (Tabela 6).

\section{CONCLUSÕES}

Os estudos de equilíbrio de adsorção mostraram que a levedura Saccharomyces cerevisiae foi efetiva como adsorvente para o íon de $\mathrm{Pb}^{2+}$. Foi possível verificar que, em condições estáticas, é necessário um tempo de contato de $48 \mathrm{~h}$ para que o equilíbrio seja alcançado. Verificou-se também que os dados de equilíbrio foram melhores ajustados pelo modelo de Langmuir. Os resultados obtidos do planejamento experimental fatorial e a análise de superfície de resposta mostraram que a quantidade adsorvida dos íons metálicos por unidade de massa de Saccharomyces cerevisiae foi influenciada pela quantidade de biomassa utilizada. Dessa forma, a interação entre a biossorção e a concentração de biomassa pode apresentar efeitos antagônicos; com isso, observou-se que maiores quantidades de biossorção, por unidade de biomassa, foram obtidas com concentrações menores de biomassa.

\section{REFERÊNCIAS}

1. Bai, R. S.; Abraham, T. E.; Bioresour. Technol. 2003, 87, 17.

2. Cruz, C. C. V.; Costa, A. C. A.; Henriques, C. A.; Luna, A. S.; Bioresour. Technol. 2003, 10, 116 .

3. Jimenez. R. S.; Dal Bosco, S. M.; Carvalho, W. A.; Quim. Nova 2004, 27, 1.

4. Shukla, A.; Zhang, Y. H.; Dubey, P.; Margrave, J. L.; Shukla, S. S.; J. Hazard. Mater. 2002, 95, 137. 
5. Petroni, S. L. G.; Pires, M. A. F.; Munita, C. S.; Quim. Nova 2000, 23, 477.

6. Kac, Y.; Tan, S.; Denizl, A.; Arica, M. Y.; Process Biochem. 2002, 37, 601.

7. Selatnia, A.; Boukazoul, A.; Kechid, N.; Bakhti, M. Z.; Chergui, A.; Kerchich, Y.; Biochem. J. 2004, 19, 127.

8. Viraraghavan, T.; Yan, G.; Bioresour. Technol. 2001, 78, 243.

9. Jianlong, W.; Xinmin, Z.; Decai, D.; Ding, Z.; Biochem. J. 2001, 87, 273.

10. Pagnanelli, F. I.; Esposito, A.; Toro, L.; Veg, L. F.; Water Resour. 2003, $37,627$.

11. Nourbakhsh, M. N.; Kiliçarslan, S.; Ilhan, S.; Ozdag, H.; Chem. Eng. J. 2002, 85, 351

12. Kiran, I.; Akar, T.; Tunali, S.; Process Biochem. 2005, 40, 3550.

13. Yan, G.; Viraraghavan, T.; Water Resour. 2003, 40, 251.

14. Basílio, M. S.; Friese, K.; de Lena, J. C.; Nalini Jr., H. A.; Roeser, H. M. P.; Quim. Nova 2005, 28, 822.
15. Bayan, Y. K.; Keskinler, B.; Cakice, A.; Levent, M.; Akay, G.; Water Resour. 2001, 35, 2191.

16. Salehizadeh, H.; Shojaosadati, S. A.; Water Res. 2003, 37, 4231.

17. Veglio, F.; Esposito, A.; Reverberi, A. P.; Process Biochem. 2003, 38, 953.

18. Dursun, A. Y.; Biochem Eng. J. 2006, 28, 187.

19. Kapoor, A.; Viraraghavan, T.; Bioresour. Technol. 1995, 53, 195.

20. Baradarajan, A.; Swaminathan, T.; Kar, S.; Bioprocess Eng. 1996, 15, 195.

21. Lo, W.; Chua, H.; Lam, K. H.; Bi, S. P.; Chemosphere 1999, 39, 2723.

22. Lisboa, M.; Rocha, S.; Greenpeace/Aspan. 1997, 16, 44.

23. Box, G. E. P.; Hunter, W. G.; Hunter, J. S.; Statistics for Experimentrs, Wiley: Canadá, 1978.

24. Ferreira, J. M.; Conrado, L .S.; Vilar, E. O.; Cavalcanti, E .B.; Alsina, O L. S; Silva, F. L. H.; Petro \& Química 2005, 278, 66.

25. Jnr, M. H. ; Spiff, A, I.; Acta Chim. Slov. 2005, 52, 174. 